\title{
Structure, mentalité, société, civilisation : les quatre linguistiques d'Antoine Meillet
}

\author{
John E. Joseph ${ }^{1 *}$ \\ ${ }^{1}$ University of Edinburgh, School of Philosophy, Psychology \& Language Sciences, Dugald Stewart \\ Building, 3 Charles Street, Edinburgh, EH8 9AD
}

\begin{abstract}
Résumé. Aujourd'hui le nom d'Antoine Meillet (1866-1936) rappelle surtout ses manuels de linguistique comparée des langues indoeuropéennes, ses études slaves et arméniennes, et sa présidence d'une " école de Paris » où seraient formés les grands linguistes de la génération suivante. Pourtant il ne faut pas oublier l'importance de ses contributions à la linguistique synchronique, y compris l'analyse de la langue française. Sa conception originale de la " grammaticalisation » dans un article de 1912, où il tire ses exemples clés du français, a démontré l'impossibilité d'une stricte séparation entre les catégories analytiques, et plus généralement entre la synchronie et la diachronie. Dans d'autres ouvrages il prétend qu'un Français conçoit un objet différemment de la façon dont un ancien Romain le concevait, à cause de la structure des grammaires latine et française ; que le passage de vocables d'un genre spécifique (tel l'argot des marins) à l'usage général est le principal moteur de l'évolution sociale d'une langue, et qu'il y a une hiérarchie quasi-féodale entre les mots selon leur origine ; qu'il n'existe en Europe que quatre «langues de civilisations », face auxquelles les autres langues seraient destinées à disparaître. Bien que Meillet ait joué un rôle cardinal dans le développement du structuralisme linguistique de la première moitié du $\mathrm{XX}^{\mathrm{e}}$ siècle, il a gardé ses distances avec le mouvement, et cette réserve, léguée à ses étudiants (à quelques exceptions près), clarifie certains aspects du caractère particulier de la linguistique française.
\end{abstract}

\begin{abstract}
Nowadays the name of Antoine Meillet (1866-1936) is mainly associated with his textbooks of Indo-European comparative linguistics, his work on Slavic languages and Armenian, and his leadership of a 'Paris school' in which the great linguists of the next generation were trained. Yet we should not lose sight of his important contributions to synchronic linguistics, including the analysis of French. With his novel concept of 'grammaticalisation' in a 1912 article, where the key examples are drawn from French, showed the impossibility of a strict separation of analytical categories, and more generally between synchrony and diachrony. In other studies he claims that a speaker of French conceives of an object differently from how an ancient Roman did, because of the structure of Latin and French grammar; that the movement of vocabulary items from a specific genre (such as the argot of sailors) into general usage is the principal driver of the social evolution of a language, and that a quasi-
\end{abstract}

\footnotetext{
*John.Joseph@ed.ac.uk
} 
feudal hierarchy exists amongst words according to their origins; that in Europe there are only four 'languages of civilisation', in the face of which other European languages are destined to disappear. Although Meillet played a cardinal role in the development of the linguistic structuralism of the first half of the 20th century, he kept a certain distance from the movement, and this reserve, which he passed on to his students (with certain exceptions), clarifies certain aspects of the particular character of French linguistics.

Notre profession a des racines cléricales, et nos discours en congrès tendent à ressembler à des sermons. C'est un tour classique du métier sacerdotal que de commencer un sermon par une confession, plaçant l'auditeur dans le rôle de confesseur. Donc je vous confesse que j'ai péché, comme étudiant de la linguistique et de son histoire, en voulant mettre chaque linguiste dans une petite boîte, étiquetée du nom d'un sous-champ et d'une démarche, même d'une innovation particulière - une seule, de préférence. En même temps j'en veux à ceux qui font de même à mon propos : car je sais bien qu'une telle réduction ne peut capturer qu'une moindre partie de ce que je vise dans mon travail. Quand ce sont des linguistes du passé que j'emballe et étiquette, c'est facile - en restant silencieux, ils semblent accepter mon jugement. Si c'est un contemporain, il peut protester, auquel cas je me dis que c'est sa fierté qui l'aveugle sur la vérité que je vois objectivement. Oui, j'ai vraiment péché : j'ai commis l'acédie, la paresse spirituelle. Ma pénitence est d'éviter désormais les étiquettes, par exemple «structuraliste », et d'essayer de faire pour les autres ce que je veux qu'ils fassent pour moi : examiner et apprécier l'intégralité de leur travail. C'est une lourde tâche quand il s'agit d'un linguiste aussi prolifique qu'Antoine Meillet. Même en survolant quatre domaines de sa vaste œuvre, je suis obligé de laisser de côté bien des aspects non-marginaux.

\section{Structure}

Aujourd'hui son nom rappelle surtout ses manuels de linguistique comparée des langues indo-européennes, ses études slaves et arméniennes, et sa présidence d'une «école de Paris » où seraient formés les grands linguistes de la génération suivante. Pourtant il ne faut pas oublier l'importance de ses contributions à la linguistique synchronique, y compris l'analyse de la langue française.

Né à Moulins en Auvergne, Meillet est allé à Paris en 1884 pour une année au Lycée Louis-le-Grand, après laquelle il est entré en Sorbonne. Les cours qui l'ont spécialement inspiré étaient ceux donnés à l'École Pratique des Hautes Études sur le gotique et le vieux haut allemand par le jeune Ferdinand de Saussure, le suppléant du professeur Michel Bréal. De l'enseignement de Saussure, Meillet a tiré les principes qui guideraient ses analyses linguistiques dorénavant, et qu'il attribuerait toujours à son maître genevois. Si on appelle cette démarche "structuraliste », il ne faut pas oublier que le mot structure n'est pas saussurien : aucune des trois instances de structure dans le Cours de linguistique générale ne réfère à une structure linguistique dans le sens structuraliste. Le terme saussurien est système, et comme Henri Meschonnic l'a souvent souligné, les systèmes sont dynamiques, alors qu'on a tendance à concevoir les structures comme rigides ${ }^{\mathrm{i}}$. Mais structure est bien un mot meilletien : quand, en 1906, il a succédé à la chaire de grammaire comparée de Michel Bréal au Collège de France, il l'a rebaptisée chaire de l'histoire et de la structure des langues indo-européennes.

L'une des conceptions structurelles de Meillet a laissé un héritage particulièrement fécond. Il a introduit le terme «grammaticalisation » pour la première fois dans un article 
de 1912, «L'évolution des formes grammaticales ». Depuis 1910, Meillet donnait une série d'articles à Scientia, une revue italienne qui visait, selon Meillet, « un public large mais curieux de science », et qui n'était pas «destiné proprement à des savants dont la linguistique est la spécialité $\rangle^{\mathrm{ii}}$. Son article de 1912, qui annonce le concept de grammaticalisation, fait partie de cette série. Dans l'histoire d'une langue ou d'une famille linguistique particulière, certains morphèmes flexionnels liés - peut-être tous - étaient, à un stade antérieur, des morphèmes lexicaux libres. Cette idée n'était pas nouvelle. Prenant un exemple familier, les désinences futures des verbes romanes coïncidaient trop étroitement avec le présent d'habere "avoir » pour que ce soit une coïncidence ; et déjà dans la grammaire espagnole de Nebrija de 1492, on pouvait lire que les futurs "synthétiques » latins, tels qu'amabo «j'aimerai », avaient été remplacés dans les langues romanes par de nouveaux futurs « analytiques », tels que aimerai, à l'origine aimer-ai «j'ai à aimer » ${ }^{\mathrm{iii}}$. Le nouveau point de vue proposé par Meillet était que toute une gamme de changements apparemment divers pouvaient être catégorisés comme étant de cette nature, constituant un processus unique qui se produit régulièrement et progressivement à travers des langues lointainement apparentées ou même non apparentées.

Les deux occurrences du terme « grammaticalisation » dans l'article de 1912 se trouvent dans la première et la dernière sections. Toutes les deux sont mises entre « scare quotes », des guillemets ironiques sans ironie, pour indiquer leur nouveauté, mais sans commentaire : ce qui suggère qu'aux yeux de Meillet le sens du mot « grammaticalisation » était évident. Voici le premier exemple iv :

Tandis que l'analogie peut renouveler le détail des formes, mais laisse le plus souvent intact le plan d'ensemble du système existant, la " grammaticalisation » de certains mots crée des formes neuves, introduit des catégories qui n'avaient pas d'expression linguistique, transforme l'ensemble du système.

Il faut, bien sûr, lire cet extrait dans son contexte. L'article commence en disant que deux processus produisent des formes grammaticales. Le premier est l'analogie. Meillet note que l'analogie était la seule explication permise par les Néogrammairiens pour des exceptions apparentes aux lois phonétiques. Leur détermination d'établir que ces lois n'admettent pas de véritables exceptions était la cause, suggère-t-il, du manque d'attention au deuxième processus, qu'il appelle «le passage de mots autonomes au rôle d'agents grammaticaux », c'est-à-dire la grammaticalisation.

Il donne l'exemple du verbe français suis. Ce verbe, dit-il, est autonome dans je suis celui qui suis, et retient toujours un degré d'autonomie dans je suis chez moi. Mais « il n'est presque plus qu'un élément grammatical dans : je suis malade, je suis maudit, et il n'est tout à fait qu'un élément grammatical dans : je suis parti, je suis allé, je me suis promené $\gg{ }^{\mathrm{v}}$. Les choses que Meillet laisse tacites sont intrigantes : est-ce du premier ou du deuxième suis qu'il parle dans le verset biblique je suis celui qui suis (Exode 3:14), ou tous les deux ? Qu'en est-il du pronom sujet clitique je, qui dans notre perspective actuelle semble être un cas plus net de grammaticalisation que suis? Son choix de mots - « a encore une certaine autonomie », « il est devenu » - implique clairement qu'au fil du temps, des éléments autonomes ont perdu leur autonomie et sont devenus «simplement» grammaticaux. L'essentiel du concept de grammaticalisation dans ce premier exposé de Meillet est qu'il entraîne une perte d'autonomie : un élément devient dépendant d'un autre.

La dépendance est clairement syntaxique dans l'exemple je suis parti, où suis est devenu un auxiliaire, mais il semble aussi être partiellement sémantique. Certains de ses exemples ultérieurs seront plus pleinement sémantiques, comme lorsqu'il dit que petits dans les petits enfants s'est grammaticalisé pour devenir « un mot accessoire », puisque, après tout, tous les enfants sont petits ${ }^{\mathrm{vi}}$. D'autres exemples font voir l'affaiblissement phonologique qui arrive souvent en parallèle avec la grammaticalisation. 
Quand Meillet dit que la grammaticalisation est un processus plus important que l'analogie parce qu'il «transforme l'ensemble du système» (ci-dessus), son souci du système dans son ensemble montre qu'il pense de façon diachronique plutôt qu'historique,

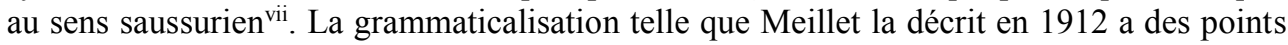
communs avec ce que Saussure avait enseigné l'année auparavant dans ses dernières leçons de linguistique générale à Genève. À propos de la limitation de l'arbitraire des signes linguistiques, Saussure a remarqué que l'évolution du latin au français a apporté «un énorme déplacement dans le sens de l'immotivé ${ }^{\text {viii }}$. Par exemple, amicus et inimicus sont évidemment apparentés en latin, mais leurs équivalents français ami et ennemi ne sont pas généralement perçus comme apparentés par les francophones. Ennemi «est rentré dans l'arbitraire absolu $»^{\mathrm{ix}}$.

Cet exemple rappelle celui que donne Meillet de la grammaticalisation du protogermanique hiu tagu «ce jour » pour devenir hiutu « aujourd'hui » en vieux haut allemand, puis heute en allemand moderne ${ }^{\mathrm{x}}$. Le mot aujourd'hui lui-même, tout comme today en anglais, sont des cas de ce type. Tous les linguistes contemporains ne les classeraient pas comme grammaticalisés, puisque leur résultat est lexical plutôt que grammatical, mais pour Meillet ce sont des exemples typiques de la grammaticalisation. Quand Saussure dit en 1911 que « tout le mouvement que représente pour la langue l'évolution peut se résumer en un va-et-vient entre la somme respective du parfaitement immotivé et du relativement motivé $\rangle^{\mathrm{xi}}$, il semble encore une fois anticiper la position de Meillet selon laquelle la grammaticalisation est le plus important des deux processus de changement à cause de ses effets sur le système dans son ensemble.

Dans le concept de grammaticalisation on voit le développement de certains thèmesclés du structuralisme de cinquante ans plus tard. Le passage d'un élément de l'état de mot indépendant à celui d'un outil grammatical est aussi son transfert du choix d'un sujet parlant qui contrôle son discours, vers une obligation imposée par la structure linguistique. Si le «sujet parlant» est un vrai sujet, au sens classique d'un agent libre, le je qui dit je pense, donc je suis, alors la perte de l'autonomie qu'on observe linguistiquement dans la grammaticalisation est en parallèle avec le changement qui s'opérait dans la pensée théorique de Meillet et ses contemporains. Le structuralisme représente un transfert de ce qu'on attribuait au sujet parlant en faveur de la grammaire, la langue. Pas un transfert total, même si aux années 1960 cela sera la caricature des structuralistes par leurs ennemis ; mais une perte d'autonomie et d'une certaine mesure de liberté est le prix de toute structure, par définition ${ }^{\mathrm{xii}}$.

\section{Mentalité}

À partir de 1920 environ, Meillet ira encore plus loin, poussé peut-être par sa lecture du Cours de linguistique générale. Saussure dit que l'anglais accorde un rôle beaucoup plus important à l'immotivé que ne fait l'allemand, puisque l'allemand indique les relations grammaticales par l'inflexion des verbes et des noms, tandis que l'anglais a retenu peu d'inflexions, et indique les relations grammaticales plutôt par la position et l'emploi d'auxiliaires et de prépositions. En ce sens, l'allemand est plus « grammatical » et incliné vers le motivé, l'anglais plus «lexical» et enclin à l'arbitraire radical. Cela devient une idée centrale dans la pensée ultérieure de Meillet. Dans une coda datée le 5 mai 1920 qu'il a ajoutée à son article de 1909 «Sur la disparition des formes simples du prétérit » pour sa réédition dans son recueil de 1921, il dit :

Le trait essentiel de la structure morphologique de l'indo-européen, et encore du latin, c'est que le mot n'existe pas indépendamment de la forme grammaticale : il n'y a pas un mot signifiant « cheval», il y a un nominatif singulier equus, un génitif singulier equi, un accusatif pluriel equos, etc. et l'on ne saurait isoler 
aucun élément signifiant «cheval» indépendamment des finales. Au contraire, dans le type moderne représenté par l'anglais, et, un peu moins bien par le français, le mot tend à exister indépendamment de tout "morphème » : quel que soit le rôle joué dans la phrase, on dit en anglais $d o g$ et en français chien, là où le latin avait une série de formes suivant les cas ${ }^{\mathrm{xiii}}$.

Bientôt, il développera des idées sur le développement psychologique des peuples indo-européens, procédant d'un stade «concret» primitif à un stade "abstrait» plus avancé. Dans son discours sur «le caractère concret du mot», lu devant la Société de Psychologie en 1922 et paru l'année suivante dans le Journal de Psychologie, Meillet écrit qu' « un Romain n'était pas capable de nommer «le loup en soi » [...]. La tendance universelle du langage, au cours de la civilisation, a été de donner au nom un caractère de plus en plus indépendant de tous ses emplois particuliers « ${ }^{\text {iv }}$. Pendant la discussion qui suit le discours, Meillet insiste sur le fait que le développement des langues doit aller du concret vers l'abstrait, et que par conséquent, « la mentalité d'un Indo-Européen diffère tout à fait d'un moderne $\gg{ }^{\mathrm{xv}}$.

Soit Meillet présentait trois visions différentes de l'évolution linguistique aux psychologues, aux sociologues et aux linguistes, soit sa propre mentalité évoluait. Ou une combinaison des deux. À mi-carrière en 1912, la grammaticalisation aussi lui apparaissait très clairement comme un passage du concret à l'abstrait. Mais la signification de ces deux mots, concret et abstrait, a été très glissante, à long terme et à court terme. En 1912 le concret paraît avoir à faire avec la précision du sens et la force avec laquelle le locuteur veut le souligner. Cette idée de force est liée à la tendance des mots, en grammaticalisant, à être réduit phonologiquement, et à la tendance des phrases à devenir des clichés, des collocations "habituelles», en route vers la grammaticalisation. Meillet dit que la signification syntaxique de l'ordre des mots résulte du même processus. Il cite la phrase Pierre bat Paul - la syntaxe a une longue histoire de violence physique - en opposition à Paul bat Pierre, ou l'ordre des mots a pris le caractère d'un morphème. Ici Meillet invoque le terme grammaticalisation pour la seconde et dernière fois ${ }^{\mathrm{xvi}}$ :
Ici, un ordre de mots devenu habituel pour quelque raison a pris le caractère de "morphème», c'est-à-dire de marque d'une catégorie grammaticale. La valeur expressive de l'ordre des mots, que l'on observe en latin, a été remplacée par une valeur grammaticale. Le phénomène est de même ordre que la " grammaticalisation » de tel ou tel mot [...].

Il semble que finalement c'est le va-et-vient entre, d'un côté, l'envie d'une expression puissante et originale, et de l'autre, l'habitude, plus le fait qu'un nombre limité de collocations «originales» sont disponibles, qui poussent le processus de grammaticalisation tel que Meillet le voyait en 1912. Il est intéressant qu'à la fin de la réédition de l'article en 1921 Meillet a ajouté une note qui recommande aux lecteurs de consulter les ouvrages sur la stylistique par Charles Bally et Leo Spitzer.

La stylistique de Bally concernait la dimension «affective» d'une langue, les éléments qui la lient directement au locuteur, une dimension d'une ampleur considérable mais qui ne représente qu'un sous-ensemble du système linguistique. Avec sa théorie de l'énonciation de 1931, Bally a voulu saisir comment une langue énonce la pensée en général, ainsi que la relation entre le locuteur et l'auditeur. Comment une langue énonce, plutôt que comment un locuteur le fait : ce n'est pas alors l'énonciation comme elle sera conçue aux années 1950 et 1960 par Lacan, Dubois et Benveniste. Chez Bally l'important est de ne pas séparer les langues de leurs locuteurs, et sa théorie de l'énonciation concerne les différences dans la façon dont la pensée est exprimée dans la langue $x$ et la langue $y$, pas entre deux locuteurs de $x$ - sa stylistique aussi se situe au niveau de la langue, à la différence de la stylistique ultérieure qui analyse le style des écrivains individuels ${ }^{\text {xvii }}$. Bally précise que sa théorie de l'énonciation est une étude des signifiés ${ }^{x v i i i}$, et que «toute 
énonciation de la pensée par la langue est conditionnée logiquement, psychologiquement et linguistiquement $»^{\mathrm{xix}}$. Bally est à la recherche des structures qui font fonctionner diverses langues différemment, en permettant aux locuteurs de s'exprimer, et d'exprimer leur relation avec leurs interlocuteurs, de sorte que certaines langues, l'allemand par exemple, sont orientées vers le locuteur, tandis que d'autres, y compris le français, sont orientées vers l'auditeur, selon son analyse.

\section{Société}

Ce n'est pas, évidemment, ce qu'on appellerait aujourd'hui la sociolinguistique ; mais c'est une analyse «sociale » dans le sens saussurien, où c'est la langue qui est le fait social, tandis que la parole comprend tout ce qui appartient à l'individu. Dans la pré-histoire de la sociolinguistique, Meillet occupe une place centrale, ayant fait partie de l'équipe éditoriale de L'année sociologique, la revue fondée par Émile Durkheim, le doyen des sociologues français, depuis la cinquième année (1900-1901). La phrase « la langue est un fait social» se lit pour la première fois dans un article de Meillet intitulé «Comment les mots changent de sens », dans 1'Année sociologique de 1905-6 $6^{\mathrm{xx}}$ Meillet, comme Saussure, emploient le terme « social » dans son sens originel de ce qui lie les gens dans une collectivité, un sens qui va subir une mutation à travers le vingtième siècle, devenant finalement son contraire. Les sociolinguistes depuis les années 1960 s'intéressent à ce qui distingue les gens les uns des autres dans « la même langue ».

L'année 1903 avait été le point culminant du débat entre Durkheim et Gabriel de Tarde sur le rôle de la psychologie dans la recherche sociologique, et de quel genre de psychologie un sociologue pourrait se servir. Durkheim et ses associés ont gagné par défaut quand Tarde est mort en 1904. Meillet s'efforçait de rester fidèle à la méthodologie de Durkheim, de façon frappante dans "Comment les mots changent de sens ». Là on trouve ses déclarations les plus claires sur les raisons pour lesquelles la langue constitue un « fait social » selon la définition de Durkheim. Meillet présente comme le moteur du changement linguistique le passage des formes des dialectes spécialisés, par exemple des argots des agriculteurs ou des marins, à la langue générale. Ce passage est, selon Meillet, essentiellement un changement social. L'article comprend des exemples de ce qu'il appellera plus tard la «grammaticalisation »: homo latin donnant l'on impersonnel du français. Mais, dit-il, "Ces cas où l'agent essentiel du changement est la forme grammaticale sont d'une espèce assez rare ${ }^{\mathrm{xxi}}$. Comme on a vu, il va changer d'avis dans les années suivantes. Meillet n'a jamais cessé de proclamer la nature sociale de la langue, mais en 1912 son attention avait commencé à s'éloigner de la méthode sociologique, peutêtre parce qu'il croyait avoir mis en place tout ce qu'il fallait à cet égard; ou bien parce que sa conception de la langue comme fait social a dépassé les bornes de la méthode durkheimienne. Je ne sais pas si, par exemple, on peut réconcilier ses idées sur la mentalité des peuples anciens avec cette assez stricte méthode sociologique.

De toute façon, ce sera l'un des étudiants de Meillet, André Martinet, en exil à Columbia University à New York après avoir été accusé de collaboration pendant la guerre, qui dirige la thèse d'Uriel Weinreich sur les langues en contact, et Weinreich à son tour dirigera la thèse de doctorat de William Labov ${ }^{x x i i}$. Il y a donc une ligne directe de Saussure et Meillet à la sociolinguistique moderne - bien que, comme je l'ai noté, la conception du social ait changé de ce qui lie les individus entre eux à ce qui les distinguent, ou plus précisément, aux variations qui marquent des sous-groupements à l'intérieur de la communauté linguistique. L'idée de la variation est présente dans le travail de Meillet sur les changements introduits avec l'entrée du vocabulaire spécialisée dans l'usage général mais c'est le fait de devenir général qui rend ces changements sociaux ; leur restriction antérieure était pour ainsi dire anti-sociale. 
Meillet était sensible à ce qu'il appelait la «valeur » des mots, qui n'est pas la valeur saussurienne, qui procède de la différence entre les signes linguistiques et fournit la base du système sémiologique. Au sujet du vocabulaire latin, Meillet dit :

\begin{abstract}
Tous les mots ne sont pas à un même niveau; il y a des mots « nobles » et des mots «roturiers». Les mots qui désignaient les idées les plus générales, comme morī et uīuere, les actes essentiels, esse et bibere, les relations de famille, pater, māter, frāter, les principaux animaux domestiques, equus, ouis, sūs, l'habitation de la famille qui était l'unité principale, domus etforēs, etc., représentent le vocabulaire de l'aristocratie indo-européenne qui s'est étendue à tout le domaine ; ces mots désignent des notions ; ils n'ont pas de valeur affective, et ils ont un minimum de valeur concrète : bos, ouis, sūs s'appliquent à la fois au mâle et à la femelle; ce sont des termes qui indiquent des biens, non des termes d'éleveurs ; de même, domus et forēs évoquent l'habitation du chef, non une construction matérielle. La valeur abstraite des mots, liée au caractère aristocratique de la langue, est un trait essentiel du vocabulaire indoeuropéen.
\end{abstract}

Je m'arrête là un moment pour dire que ce que Meillet dit ici est stupéfiant pour un anglophone. C'est peut-être différent pour un romanophone ; mais moi, j'attendais que Meillet classe les mots cités, surtout equus, bos, ouis et sūs, mais aussi esse et bibere, comme « roturiers ». En anglais, leurs équivalents horse, ox, sheep, pig, eat, drink sont germaniques, et depuis 1066 les roturiers chez nous sont les gens anglo-saxons et les mots anglo-saxons. C'est le serf anglo-saxon qui soigne les oxen, sheep et pigs dont son maitre anglo-normand va manger le beef, mutton, et pork. La division du travail est différente dans les langues romanes, et il n'est pas déraisonnable de supposer qu'un noble pense au bétail comme propriété, et laisse au paysan qui s'en occupe d'assurer leur reproduction. Meillet continue :

Mais il y avait aussi des mots de caractère "populaire », reconnaissables à beaucoup de traits, vocalisme radical $\breve{a}$, gémination de consonnes intérieures, etc.; ces mots ont souvent une valeur affective, souvent un caractère technique. La plupart du temps, au moins sous les formes qu'ils ont en latin, les mots de ce genre n'ont de correspondants que dans peu de langues ; beaucoup n'en ont pas. Le vocabulaire « populaire » est aussi instable que le vocabulaire aristocratique est permanent ${ }^{\mathrm{xxiii}}$.

Encore une fois, j'aurais prévu l'inverse : que le vocabulaire populaire est le plus stable. À plusieurs reprises Meillet met son analyse en opposition à celle de « M. Vossler et son école », ces idéalistes qui prenait leur inspiration de Benedetto Croce. Meillet rejette vaguement l'importance accordée par Vossler à la perte du futur synthétique entre le latin et les langues romanes, et ce que cette perte est censée signifier quant à un changement de «mentalité ». Tout de même, les déductions de Meillet ne sont pas aussi différentes de celles de Vossler que sa rhétorique le ferait paraître. À propos du temps et de l'aspect dans le développement du vieux latin au latin classique, Meillet écrit :

La catégorie du temps est rationnelle, et celle de l' "aspect » verbal, exprimant le degré d'achèvement $\mathrm{du}$ procès, est relativement concrète. On conçoit donc que, avec le développement d'une civilisation de caractère intellectuel, où la pensée des hommes dirigeants prend un tour philosophique exact, où les enfants et les jeunes gens se forment dans les écoles, la notion de « temps » prenne le pas sur la notion d' « aspect »xiv.

Encore une fois il s'agit de la sociolinguistique : Meillet invoque la pensée des hommes dirigeants, la formation dans les écoles, pour expliquer l'origine du temps rationnel, c'est-àdire abstrait, non pas remplaçant entièrement l'aspect concret, mais prenant la priorité sur lui, tout comme les hommes dirigeants, les nobles, ont la préséance sur les roturiers. 
Le développement de la linguistique structurale est alors une histoire de différence, de se différencier d'une approche perçue comme trop psychologique et s'occupant insuffisamment de la description soigneuse des faits de structure avant d'en offrir des explications, ce qui rend douteuse la durabilité de ces explications. Aux yeux de Meillet « l'idéalisme » de Vossler est trop, enfin, idéaliste, tandis que sa propre approche est mieux ancrée dans les faits « concrets ». Historiquement, Meillet croit que le passage du concret à l'abstrait représente un progrès, et méthodologiquement aussi, sa propre analyse procède dans le même sens, déduisant des explications abstraites de son examen détaillé des phénomènes. Mais une abstraction qu'on affirme sans la déduire de cette façon, comme Meillet le trouve chez Vossler, risque d'être la fantaisie de l'analyste, donc de ne fournir aucun progrès ; d'être, non pas une noblesse destinée à régner sur ceux dont la mentalité est concrète, mais une tyrannie à laquelle les vrais nobles doivent résister.

\section{Civilisation}

Cela nous amène à la dernière des quatre linguistiques meilletiennes qui figurent dans mon survol. En 1918 Meillet a publié un livre sur Les langues de l'Europe nouvelle. Ce livre reflète la perception croissante d'une diminution de la diversité linguistique, une homogénéisation qui menace les petites langues européennes telles que le finnois et le hongrois, mais aussi les langues scandinaves, l'italien, l'espagnol et le portugais, le néerlandais, les langues baltiques et balkanes. Toutes ces langues sont condamnées par une sorte de sélection naturelle qui limiterait le nombre de «langues de civilisation » en Europe. Aujourd'hui, on voit que cette perception n'était pas illusoire, malgré les faiblesses du raisonnement et de la prédiction. Dans la «vieille» Europe, chaque village, chaque hameau avait son dialecte nettement idiosyncrasique - sa propre langue, à vrai dire - et seule une petite élite parlait la langue nationale, cette création " artificielle » de l'époque moderne. Mais plusieurs facteurs - le début de l'éducation universelle, par étapes, commençant dans la plupart des pays au dernier tiers du dix-neuvième siècle ; l'amélioration de ce qu'on appelait la « communication » par la construction de canaux, de chemins de fer, des routes adaptées aux automobiles, et dans un autre sens par les journaux, le phonographe et bientôt la radio ; la formation en temps de guerre de bataillons d'hommes de différentes régions - tout cela a contribué à la métamorphose longue et lente d'une Europe «nouvelle» où les gens dans un pays, à part les immigrant récents, peuvent généralement converser. Pas seulement les clercs parlant latin et les aristocrates parlant français, mais tous parlant la langue nationale.

La théorie la plus extrême basée sur cette perception d'une homogénéisation était le stadialisme de Marr. Le livre de Meillet est loin de cela, mais il prévoit que, pas seulement les dialectes, mais des langues entières ne vont pas survivre les changements en cours. Le hongrois, par exemple, est condamné à disparaître, à cause de son petit nombre de locuteurs, son isolation typologique vis-à-vis des langues qui l'entourent géographiquement, sa «structure compliquée» et le fait d'être le véhicule d'une civilisation que Meillet ne considérait pas comme « originelle ${ }^{\mathrm{xxv}}$. Selon lui,

Les petites langues nationales sont une étape par où passent les peuples peu cultivés pour s'approcher de la civilisation universelle. Mais la multiplicité des petites langues actuellement employés en Europe, incommode dès maintenant, prépare des crises qu'il sera difficile de résoudre, car elle va contre les tendances générales de la civilisation ${ }^{\mathrm{xxvi}}$.

Ah, ce sacré optimisme pour la mission civilisatrice ! - nous qui sommes d'un certain âge nous en souvenons vaguement aux jours de notre enfance. Il n'est pas surprenant que ces opinions de Meillet aient été très mal reçus en Hongroie, où se trouvait à ce moment l'un de 
ses étudiants, Aurélien Sauvageot. Meillet l'avait envoyé à Budapest pour apprendre le hongrois avec Gombozc, un philologue férocement nationaliste, un trait commun aux philologues hongrois de l'époque. Meillet ignorait que les faits mêmes qu'il citait l'isolation, le sentiment d'être assiégé, auxquels on peut ajouter la condescendance des Parisiens, que tout le monde connaissait, y compris les Parisiens eux-mêmes - tout cela nourrissait la ferveur culturelle pour maintenir et renforcer la langue nationale.

Naturellement, le jeune Sauvageot a attrapé la fièvre. Dans les cercles qu'il fréquentait à Budapest, il se dissociait des avis embarrassants de son maître. Mais il lui est resté fidèle, et dans ses mémoires de l'époque il écrivait :

\begin{abstract}
Meillet avait sa conception très personnelle du rôle des langues dans l'élaboration de l'avenir de la civilisation, qu'il se refusait de discuter de l'avenir des langues. Pour lui, la civilisation moderne était d'expression indo-européenne et, dans la mesure où elle tendait à s'universaliser, elle serait exprimée par les langues qu'il appelait «langues de civilisation ». Pour lui, les langues en question étaient celles qui avaient la plus grande expansion et étaient porteuses des civilisations les plus développées. En Europe, il s'agissait essentiellement du français, de l'anglais et de l'allemand du côté occidental, du russe à l'est [...]. Avec de telles prémisses, Meillet ne pouvait aboutir qu'à une conclusion : l'Europe n'avait pas intérêt à accentuer son morcellement linguistique. La multiplication des langues secondaires, les «petites » langues, lui serait fatale. C'était dépenser à tort trop d'énergie que de créer dans chaque État indépendant une langue de civilisation de plein exercice. [...] Selon lui, il était absurde de vouloir exprimer par des décalques une même pensée commune à l'Europe que cette multiplicité des variantes ainsi créées risquait de désintégrer plus ou moins complètement. Il redoutait une «balkanisation» de l'Europe. Il souhaitait voir les nations secondaires choisir de s'exprimer selon deux registres : celui de leur propre langue, pour les besoins ordinaires de la vie, et celui d'une langue de civilisation, pour tout ce qui s'élevait au-dessus des contingences locales ${ }^{\mathrm{xxvii}}$.
\end{abstract}

Dans la dyade que Patrick Sériot (2014) décrit entre une approche «romantique» du langage, caractéristique de la philosophie et de la politique allemande, et une approche «jacobine» qui a été son homologue française, Meillet, en appelant à ce bilinguisme fonctionnel, gardait un pied dans chaque camp. Mais il n'en était pas ainsi pour les nationalistes dévoués aux «petites » langues, qui ne tenaient pas bien à ce que leur idiome national soit relégué à «l'ordinaire » et au «local», ou que leur culture nationale soit traitée comme non «originale». Ce n'était pas seulement pour eux une question de chauvinisme ou de nostalgie, mais de survie pratique. Ils ont reconnu, qu'ils puissent ou non l'admettre publiquement, que Meillet avait raison au sujet des forces historiques qui conspiraient contre eux. La Grande Guerre l'avait clairement montré ; et les idées romantiques sur le lien entre la langue et la façon de penser d'un peuple, l'unifiant et lui donnant un droit naturel à l'autonomie politique, jouaient un rôle très important lorsqu'il s'agissait de redessiner la carte de l'Europe après la guerre. Le côté obscur de cette vision romantique était ses implications pour les nations ethniques qui n'avaient pas de base géographique unifiée, en particulier les Juifs et les Roms.

Cela ne veut pas dire que les nationalistes linguistiques en Hongrie et ailleurs qui ont trouvé le côté jacobin de Meillet insultant et oppressif étaient des chiens de course des nazis. Qui aurait pu prévoir la logique tordue qui conduirait du nationalisme linguistique aux camps de concentration, même si, rétrospectivement, le chemin semble inévitable ? C'est un cas où nous devons être disciplinés quant à l'application de la méthodologie historique. La dyade romano-jacobine n'est pas en noir et blanc. Elle définit un paradoxe, qui chevauche la dichotomie discutée depuis plusieurs décennies par le philosophe canadien 
Charles Taylor, qui le met au centre de son livre récent intitulé L'animal langage. Taylor contraste ce qu'il appelle le $\mathrm{H}-\mathrm{L}-\mathrm{C}$ et le $\mathrm{H}-\mathrm{H}-\mathrm{H}$, où le $\mathrm{H}-\mathrm{L}-\mathrm{C}$ est la perspective des Lumières héritée par les philosophes analytiques de Hobbes, Locke et Condillac. Le H-H-H est le point de vue «romantique » de Hamann, Herder et Humboldt, sur lequel Taylor s'efforce d'attirer l'attention des philosophes. Le jacobinisme de Sériot incarne la perspective H-L-C.

Les «besoins de la vie ordinaires » auxquels Meillet attribuerait les langues nationales sont avant tout des besoins corporels, alors que ce qui passe «au-delà des contingences locales " sera élevé, abstrait, conceptuel, mental plutôt que physique. Ce qui dépasse les contingences locales sera nécessairement social à grande échelle, alors que les "besoins ordinaires " sont des besoins individuels qui, s'ils sont sociaux, le sont dans un sens que Meillet aurait considéré comme trivial. Il y a ici un écho de la distinction langue-parole de Saussure, dans laquelle la langue est le fait social par excellence.

Contrairement à Meillet en 1918, Sauvageot était convaincu que les langues ne sont pas destinées à prospérer ou à dépérir par des tendances culturelles et politiques, des structures qu'un écrivain, un linguiste, un universitaire sont incapables de surmonter. Peutêtre va-t-il changer d'avis : en 1934, Sauvageot et Meillet étaient parvenus à un rapprochement suffisant sur cette question pour qu'ils co-écrivent un article sur «le bilinguisme des hommes cultivés ", où Meillet fournit le cadre théorique que Sauvageot

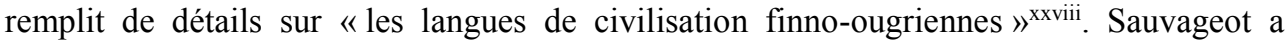
résumé l'article ainsi :

Les langues sont en partie fabriquées de toutes pièces et consciemment par les élites qui les parlent et qui s'en servent comme instrument pour l'expression de leur pensée. Le bilinguisme des hommes cultivés joue ici un rôle essentiel. Faute de le reconnaitre et d'en déceler l'action, on risque de s'abuser complètement sur l'histoire des langues. L'histoire linguistique tend donc de plus en plus à se confondre avec l'histoire de la civilisation elle-même ${ }^{\text {xxix }}$.

Meillet a construit son cadre théorique avec sa connaissance d'un grand nombre de langues, anciennes et modernes ; mais c'est surement sa langue maternelle qui l'a informé plus qu'aucune autre.

L'article de 1934 indique la direction dans laquelle Sauvageot dirigera ses intérêts futurs, pour la langue comme véhicule d'expression de la pensée que fabriquent les individus, dont une élite, cultivée et bilingue, pourront « élaborer » une nouvelle langue sur le patron d'une langue classique ou internationale. Selon Saussure, aucun individu ne peut changer la langue directement. Mon interprétation de l'article de 1934 est que Sauvageot y rejette carrément ce principe saussurien d'immutabilité, alors que Meillet le contourne, en prenant les changements introduits par les hommes cultivés comme des actes individus de parole qui auraient toujours besoin de la sanction sociale avant d'entrer dans le système de la langue. Cela ne transgresse pas les limites du saussurisme que Meillet avait été l'un des premiers à formuler, trente ans avant.

\section{Conclusion}

$\mathrm{Au}$ début $\mathrm{j}$ 'ai indiqué mon intention de ne pas réduire Meillet à une formule, à ne pas emballer et étiqueter son œuvre, mais de l'apprécier dans son amplitude. C'est maintenant l'heure de la pire tentation, parce que pour bien conclure un discours il faut nouer les fils, fournir un terminus qui aura valu le voyage. Je termine alors par une seconde confession : que je ne sais pas conclure sans réduire. Le voyage a été le but, pas le moyen de l'atteindre. Et voilà la morale de la fable : ne soyons pas satisfaits de connaître « la linguistique » d'une époque, car il y en a toujours plusieurs, souvent même chez un seul linguiste. L'entrée pour Meillet dans l'Encyclopédie universalis dit que «Meillet, soucieux de la plus grande 
exactitude, s'est toujours méfié des reconstitutions trop hâtives ». Sans prétendre avoir atteint la plus grande exactitude, j'espère avoir appliqué un esprit meilletien à cette reconstitution de quelques aspects peu appréciés de sa pensée linguistique.

\section{Références}

Bally, Charles. 1932. Linguistique générale et linguistique française. Paris, Ernest Leroux.

Benveniste, Émile. 1970. L'appareil formel de l'énonciation. Langages 17.12-18. (Réimpr. in Benveniste, Problèmes de linguistique générale, t. 2, 79-88, Paris, Gallimard, 1974.).

DeLancey, Scott. 2004. Grammaticalization: From Syntax to Morphology. In : Geert Booij, Christian Lehmann, Joachim Mugdan \& Stavros Skopeteas (Éds, avec la collaboration de Wolfgang Kesselheim), Morphology : An International Handbook on Inflection and Word-formation, t. 2, 1590-1599. Berlin \& New York, Walter de Gruyter.

Dubois, Jean. 1969. Énoncé et énonciation. Langages 13.100-110.

E[1liott], A. M[arshall]. 1892. Lebrija and the Romance Future Tense. Modern Language Notes 7/8: 485-488.

Hopper, Paul J. \& Elizabeth C. Traugott. 2013. Grammaticalization. $2^{\mathrm{e}}$ éd. Cambridge, Cambridge University Press.

Joseph, John E. 2012. Saussure. Oxford, Oxford University Press.

Joseph, John E. 2014. The Wolf in Itself : The Uses of Enchantment in the Development of Modern Linguistics. In : Rens Bod, Jaap Maat \& Thijs Weststeijn (Éds), The Making of the Humanities, III, 81-96. Amsterdam, University of Amsterdam Press; Chicago \& London, University of Chicago Press.

Joseph, John E. 2016. Divided Allegiance : Martinet's Preface to Weinreich's Languages in Contact (1953). Historiographia Linguistica 43/3.343-362.

Joseph, John E. 2019. The Resistant Embrace of Formalism in the Work of Émile Benveniste and Aurélien Sauvageot. In : James McElvenny (Éd.), Form and Formalism in Linguistics, 141-174. Berlin, Language Science Press.

Joseph, John E. 2020. The Agency of Habitus : Bourdieu and Language at the Conjunction of Marxism, Phenomenology and Structuralism. Language \& Communication 71.108-122.

Joseph, John E. Sous presse. Making Grammars Concrete Again : Aurélien Sauvageot's Esquisses of Finnish and Hungarian. In : Raf van Rooy (Éd.), The Architecture of Grammars over Time. Leuven, Peeters.

Lacan, Jacques. 2013. Le séminaire, livre VI : Le désir et son interprétation (1958-1959). JacquesAlain Miller (Éd.). Paris, La Martinière.

Meillet, Antoine. 1905/6. Comment les mots changent de sens. Année sociologique 9.1-38. (Réimpr. in Meillet $1921: 231-271)$.

Meillet, Antoine. 1909. Sur la disparition des formes simples du préterit. Germanische-Romanische Monatsschrift 1.521-526. (Reimpr. in Meillet 1921 : 149-158.)

Meillet, Antoine. 1912. L’évolution des formes grammaticales. Scientia 12.384-400. (Réimpr. in Meillet 1921 : 130-148.)

Meillet, Antoine. 1918. Les langues dans l'Europe nouvelle. Paris, Payot.

Meillet, Antoine \& Aurélien Sauvageot. 1934. Le bilinguisme des hommes cultivés. Conférences de l'Institut de linguistique de l'Université de Paris 2.5-14.

Meillet, Antoine. 1921. Linguistique historique et linguistique générale, t. 1. Paris, Champion.

Meillet, Antoine. 1923. Le caractère concret du mot. Journal de Psychologie Normale et Pathologique 20.246-258. (Réimpr. in Meillet 1936 : 9-13.)

Meillet, Antoine. 1932. $2^{\mathrm{e}}$ Avertissement à Alfred Ernout \& Antoine Meillet, Dictionnaire étymologique de la langue latine: Histoire des mots. Paris, Klincksieck.

Meillet, Antoine. 1936. Linguistique historique et linguistique générale, t. 2. Paris, Champion.

Meschonnic, Henri. 2009. Traduire, et la Bible, dans la théorie du langage et de la société. Nouvelle revue d'esthétique 3.19-26.

Perrot, Jean. 2009. La carrière et l'œuvre d'Aurélien Sauvageot : engagement et retenue dans les options linguistiques. Études finno-ougriennes 41.9-25. 
Saussure, Ferdinand de. 1916. Cours de linguistique générale. Charles Bally \& Albert Sechehaye (Éds), avec la collaboration of Albert Riedlinger. Paris \& Lausanne, Payot. ( $2^{\mathrm{e}}$ éd. 1922; éds. ultérieures presque inchangées.)

Saussure, Ferdinand de, \& Émile Constantin. 2005. Saussure, Notes préparatoires pour le cours de linguistique générale 1910-1911 ; Constantin, Linguistique générale, cours de M. le professeur de Saussure 1910-1911. Daniele Gambarara \& Claudia Mejía Quijano (Éds). Cahiers Ferdinand de Saussure 58.71-290.

Sériot, Patrick. 2014. Language and Nation : Two models. In : Virve-Anneli Vihman \& Kristiina Praakli (Éds), Negotiating Linguistic Identity : Language and Belonging in Europe, 255-274. Oxford \& Bern, Peter Lang.

Smith, Andrew D. M., Graeme Trousdale \& Richard Waltereit (Éds). 2015. New Directions in Grammaticalization Research. Amsterdam \& Philadelphia, John Benjamins.

Taylor, Charles. 2019. L'animal langage : La compétence linguistique humaine. Montréal, Boréal.

Weinreich, Uriel. 1953. Languages in Contact : Findings and Problems. New York, Publications of the Linguistic Circle of New York.

\footnotetext{
${ }^{\mathrm{i}}$ Voir par ex. Meschonnic (2009).

ii Meillet (1921 : vii).

iii Voir E[1liott] (1892), et sur la grammaticalisation en général, Hopper \& Traugott (2003), Smith et al. éds (2015).

iv Meillet (1912: 387 [1921: 133])

${ }^{v}$ Meillet (1912: $\left.385[1921: 131]\right)$

${ }^{v i}$ Meillet (1912: 388 [1921: 134]). Cela réfère à un autre passage biblique : laissez venir à moi les petits enfants (Luc 18:16). Cet exemple permet à Meillet d'expliquer comment le causatif laisser est devenu « en quelque mesure, un auxiliaire ». DeLancey (2004: 1590) remarque qu'on y voit la conception meilletien de la grammaticalisation comme " a gradual process rather than a sudden categorial shift». Meillet admet aussi que chacun des mots laisser et petit peut être un « mot principal »en d'autres contextes, telle que «laissez cela» et « apportez le petit paquet (et non pas le gros paquet qui est à côté) ».

vii Voir Joseph (2012: 383, 551-552)

viii Saussure et Constantin (2005: 233); cf. Saussure (1922 [1916] : 184).

ix Ibid.

${ }^{x}$ Meillet (1912: 392 [1921:138-139]).

xi Saussure \& Constantin (2005: 232-233).

xii Sur ces questions complexes voir aussi Joseph (2020).

xiii Meillet (1921 : 156-157).

xiv Meillet (1936 [1923] : 11-13).

xv Ibid., 17; voir aussi Joseph (2014).

xvi Meillet (1912: 400 [1921:148]).

xvii Bally tient pour acquis que les locuteurs individuels diffèrent dans leur production de la parole. La manière dont ils formulent des énoncés clairs ou peu clairs, intéressants ou inintéressants pour leurs auditeurs, serait quelque chose à étudier dans le cadre de la linguistique de la parole projetée par Saussure.

xviii Bally $(1932: 25)$.

xix Bally $(1932: 31)$.

xx Meillet (1904/5). La couverture du numéro dit « Neuvième année, 1904-5 », suivie de la date de publication 1906 ; les pages internes du numéro disent «L'année sociologique, 1905-1906 ».

xxi Meillet (1905/6:10 [1921:240-241].

xxii Weinreich (1953) est basé sur sa thèse de doctorat. Pour plus d'informations voir Joseph (2016).

xxiii Meillet (1932).

xxiv Ibid., pp. 270-271.

xxv Perrot (2009: 11).

xxvi Meillet (1918: 279 .

xxvii Sauvageot (2013:209-210). Voir aussi Joseph (2019 et sous press).

xxviii Meillet \& Sauvageot (1934).

xxix Cité par Perrot (2009: 12), entre guillemets mais sans en spécifier la source.
} 